\title{
Empreendedorismo em enfermagem: contribuição ao objetivo de desenvolvimento sustentável Saúde e Bem-Estar
}

\author{
Entrepreneurship in Nursing: contribution to the Health and Well-being Sustainable Development Goal \\ Emprendimiento en Enfermería: contribución al objetivo de desarrollo sostenible Salud y Bienestar
}

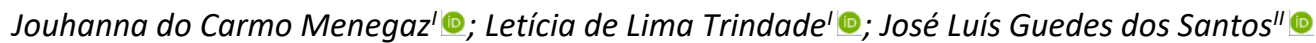

'Universidade do Estado de Santa Catarina, Chapecó, Santa Catarina, Brasil;

"Universidade Federal de Santa Catarina, Florianópolis, Santa Catarina, Brasil

\begin{abstract}
RESUMO
Objetivo: refletir sobre a relação entre o empreendedorismo de Enfermagem e as metas do Objetivo de Desenvolvimento Sustentável (ODS) no Saúde e Bem-Estar. Conteúdo: apresentam-se conceito e tipologias de empreendedorismo empreendedorismo social, empreendedorismo empresarial e intraempreendedorismo - com exemplificação de atividades da Enfermagem, bem como as metas do objetivo 3, Saúde e Bem-Estar, com foco central em refletir acerca das potenciais contribuições da atuação empreendedora no âmbito de cada tipologia. Conclusão: a atuação empreendedora tem potencial, inclusive em interface com as metas dos objetivos de desenvolvimento sustentável 8 e 4 . Porém, uma vez circunscrita no contexto dos desafios do sistema de saúde brasileiro, necessita de fomento e educação.
\end{abstract}

Descritores: Educação em Enfermagem; Mercado de Trabalho; Empreendedorismo; Papel do Profissional de Enfermagem.

\begin{abstract}
Objective: to reflect on the relationship between Nursing entrepreneurship and the Health and Wellness Sustainable Development Goal (SDG). Content: the concept and typologies of entrepreneurship (social entrepreneurship, business entrepreneurship and intrapreneurship) were stated and exemplified by Nursing activities, as were the objectives of SDG 3, Health and Wellness, focusing centrally on thinking about the potential for entrepreneurial action within each typology. Conclusion: entrepreneurial action has potential, including at the interface with the objectives of SDGs 8 and 4. However, circumscribed as it is by the challenges facing the Brazilian health system, entrepreneurship needs promotion and education. Descriptors: Education, Nursing; Job Market; Entrepreneurship; Nurse's Role.
\end{abstract}

\section{RESUMEN}

Objetivo: reflexionar sobre la relación entre el emprendimiento en Enfermería y los retos del Objetivo de Desarrollo Sostenible (ODS) en Salud y Bienestar. Contenido: se presentan concepto y tipologías de emprendimiento - emprendimiento social, emprendimiento empresarial e intraemprendimiento - con ejemplos de actividades de Enfermería, así como los retos del objetivo 3, Salud y Bienestar, cuyo enfoque central es reflexionar sobre las potenciales contribuciones de la acción emprendedora en el ámbito de cada tipología. Conclusión: la acción emprendedora tiene potencial, incluso en la interfaz con los retos de los objetivos de desarrollo sostenible 8 y 4 . Sin embargo, una vez circunscrita en el contexto de los desafíos del sistema de salud brasileño, necesita incentivo y educación.

Descriptores: Educación en Enfermería; Mercado de Trabajo; Emprendimiento; Rol de la Enfermera.

\section{INTRODUÇÃO}

O Brasil é um país que destaca em sua Constituição a saúde como direito e dever do Estado e para isso instituiu um sistema que tem como princípio o acesso universal. Como Estado Membro das Nações Unidas comprometeu-se em atingir a cobertura universal de saúde (CUS) até 2030, como parte dos Objetivos do Desenvolvimento Sustentável (ODS). Estes foram ratificados em 193 países em 2015, trazendo uma agenda global composta por 17 Objetivos e 169 metas $^{1}$, entre os quais destaca-se, por sua relação com o trabalho de Enfermagem, o ODS 3, Saúde e Bem-Estar.

As crescentes e diversificadas necessidades de saúde da população, o tempo e o custo de acesso a certos serviços são alguns dos principais entraves de acesso e que também tem impacto no mercado de trabalho em diferentes países ${ }^{2}$ e marcadamente no Brasil ${ }^{3-4}$, fazendo surgir um mercado paralelo, para além das operadoras de planos de saúde e da categoria médica, mercado este que tem buscado suprir demandas reprimidas do Estado, mas cuja relação não se dá de forma pautada nos princípios do Sistema Único de Saúde (SUS).

Contudo, frente às controvérsias do conceito de cobertura universal, em particular nas Américas, onde há discussões sobre adotar uma estratégia de saúde universal como elemento conciliador de paradigmas distintos ${ }^{5}$, observa-se a permanência do desafio de promover o acesso da população a serviços e ações, desde a promoção da saúde até à prevenção, tratamento, reabilitação e cuidados paliativos. Ainda, em um cenário de pandemia e com necessidade de preparo para o póspandemia, com projeções de crise econômica e restrições orçamentárias ${ }^{3}$ este desafio tenderá a aumentar. 
Neste sentido, atingir a CUS no Brasil até 2030 deve passar pelo investimento na Enfermagem, não apenas restrita aos que atuam em estabelecimentos ou posições assistenciais mais tradicionais de cuidados, como unidades básicas de saúde e hospitais ${ }^{6}$, nem somente como empregada, mas nos que possuem atuação empreendedora, com potencial e oportunidades para explorar novos espaços sociais.

A Organização Mundial da Saúde (OMS) e a Organização Pan-Americana da Saúde (OPAS) têm convocado os países a investir em profissionais de enfermagem para avançarem rumo à CUS, investimento este que por vezes tem sido compreendido somente no contexto da esfera pública, o que é limitado para um sistema de saúde como o brasileiro, que paulatinamente admite limitações ${ }^{7}$, destacando nos planos nacionais e estaduais de saúde a necessidade de ampliação da cobertura de acesso frente a dados epidemiológicos que indiciam que as necessidades de saúde das pessoas não estão sendo plenamente atendidas, e que, inclusive, por conta destas, permite a participação da iniciativa privada de forma suplementar.

Sendo assim, a categoria profissional pode atuar em distintas frentes, possuindo potencial para empreender, no caso dos enfermeiros, também como profissionais liberais, em clínicas e consultórios, o que a médio e longo prazo pode ampliar a rede assistencial. Isto posto, propõe-se refletir sobre a relação entre o empreendedorismo de Enfermagem e as metas do objetivo de Desenvolvimento Sustentável Saúde e Bem-Estar.

\section{CONTEÚDO}

\section{Empreendedorismo de Enfermagem}

Na literatura há muitas definições de empreendedorismo focalizando diferentes aspectos, conforme a área do conhecimento. Porém, de modo geral, pode-se dizer que há duas conceituações principais. Uma, mais antiga, sinaliza a compreensão de que empreender se trata de carreira, ocupação, atuação autônoma e a posse de um negócio, gerando valor, produtos e processos advindos de atividades econômicas; e outra, mais recente, que compreende empreender como comportamento caracterizado pela identificação ou criação de oportunidades ${ }^{8}$.

De forma similar, revisão sobre o conceito e tipologias na Enfermagem identificou na produção a predominância da compreensão comportamental, bem como três tipologias: empreendedorismo social, empreendedorismo empresarial e intraempreendedorismo, nesta ordem de relevância ${ }^{9}$.

O empreendedorismo social caracteriza-se pela preocupação com o desenvolvimento e transformação da sociedade, relacionando-se com ideias de sustentabilidade, emancipação e prestação de serviços a pessoas, grupos ou comunidades em situação de vulnerabilidade, sendo o SUS reconhecido como espaço fértil para difusão de práticas. Na Enfermagem, isto se caracteriza, por exemplo, em realizar consultas, visitas e consultorias de enfermagem, além de atividades comunitárias, a exemplo dos exames clínicos de enfermagem gratuitos ${ }^{10}$.

Já o empreendedorismo empresarial caracteriza-se pela atuação autônoma do enfermeiro, como profissional liberal ou proprietário de um negócio. O enfermeiro identifica oportunidades no sistema de saúde e oferta produtos e serviços no âmbito de sua competência ética, legal e técnica que são compreendidos como formas potentes de reforma no próprio sistema. Nessa direção tem-se a emergência de ações e serviços à nível de atenção primária e secundária de forma integrada e planejada, para oferta de serviços de enfermagem em clínicas e consultórios direcionados à saúde humana em seu ciclo vital.

Por fim, o intraempreendedorismo, também chamado de empreendedorismo coorporativo, se caracteriza pelo desenvolvimento de ações empreendedoras em organizações públicas ou privadas em que o pessoal de enfermagem é servidor/contratado sendo objeto de ação seu próprio cenário de atuação ${ }^{9}$. Nessa vertente o empreendedor empreende em organizações já existentes e busca soluções para questões do cotidiano. A presença dos enfermeiros em clínicas integradas e atuação em equipes multiprofissionais que ofertam atendimento domiciliar, são alguns dos exemplos da possibilidade de intraempreendedorismo.

Independentemente de compreender-se o empreendedorismo pela via econômica ou comportamental, de compreender-se que a categoria possui tropismo para o social, empresarial ou intraempreendedorismo, deve se admitir o fato de que a Enfermagem, especialmente o enfermeiro, desde muito tempo possui prerrogativa para empreender seja como empregado, profissional liberal ou dono de seu negócio.

\section{Contribuições do empreendedorismo de Enfermagem para o ODS 3}

O ODS 3 volta-se à Saúde e Bem-Estar, que intenciona garantir o acesso à saúde de qualidade e promover o bemestar para todos, em todas as idades, com nove metas. No conjunto das metas, sintetizam-se temáticas que tem interface direta com o trabalho da Enfermagem, em particular no campo assistencial e educativo, no âmbito de ações primárias, entre elas a redução da mortalidade materna e infantil; por acidentes; o enfrentamento na direção da erradicação e combate de diferentes doenças epidêmicas e transmissíveis; a promoção da saúde mental e o bem-estar; 
a prevenção e o tratamento do abuso de drogas lícitas e ilícitas; por exposição a produtos químicos perigosos e à contaminação e poluição do ar e água do solo e de acesso universal a serviços de saúde sexual e reprodutiva e cobertura universal a serviços essenciais, na perspectiva da proteção ao risco financeiro ${ }^{1}$.

Há ainda no ODS 3 metas relacionadas à pesquisa, desenvolvimento e acesso a vacinas e medicamentos e formação e retenção do pessoal de saúde nos países em desenvolvimento, que também relacionada ao ODS 4, referente ao trabalho, e ao ODS 8, referente a educação ${ }^{1}$.

Há duas décadas a ideia de saúde global que sustenta a proposição dos ODS, não existia ${ }^{11}$. Este novo paradigma requer da Enfermagem, em especial dos enfermeiros compreensão que negocie a preocupação com o planeta, que transcenda o território, mas que ao mesmo tempo os conduza a ações concretas onde estão. Para tanto, é relevante caracterizar o mercado de trabalho da enfermagem para que se possa relacionar o ODS 3 e os espaços potenciais e latentes para empreender no contexto da tipologia apresentada.

Analisa-se em estudo ${ }^{12}$ a inserção da enfermagem nos setores público, privado e filantrópico, concluindo que a categoria está ainda bastante institucionalizada e que o setor público é seu maior empregador. Em cada setor os profissionais estão empregados em estabelecimentos que o caracterizam majoritariamente. No público predominam empregos em estabelecimentos que oferecem assistência nas especialidades básicas como postos e centros de saúde, no setor privado em clínicas, policlínicas e ambulatórios e no setor filantrópico em hospitais.

A atuação empreendedora da enfermagem, no âmbito de cada tipologia, torna-se fundamental, especialmente por possuir características peculiares, todas relevantes a saúde global-local uma vez que, no que tange as necessidades de saúde, se direcionam a estratos distintos da população, contribuindo, sobretudo, para a ampliação do acesso em cada um destes estabelecimentos e também criando oportunidades de atuação em novos espaços e ofertando serviços dentro de sua competência técnica e legal.

$\mathrm{Na}$ atuação empreendedora social todos os profissionais de enfermagem são atores em potencial, distinguindose mais pela característica da atuação empreendedora, voltada a articulação entre o social e a saúde, contribuindo para com o viver saudável. Trata-se de uma atuação empreendedora que leva em conta o contexto da pessoa, que transcende os espaços e processos tradicionais de cuidado ${ }^{13}$, contribuindo, portanto, para a ampliação do acesso a saúde de pessoas ou grupos com dificuldades de acesso aos serviços de saúde, em situação de vulnerabilidade ou até invisibilidade social. Assim sendo, a realização de ações de promoção e prevenção a saúde no âmbito da competência ético-legal de enfermagem, como, por exemplo, as consultas de enfermagem fora de estabelecimentos assistenciais, sustentadas em teorias de enfermagem.

Além da atenção a ampliação do acesso a grupos, na direção das metas do ODS 3 há de se buscar a ampliação dos serviços, pois a oferta atual parece incipiente para a amplitude deste objetivo. Assim, tem-se na atuação empreendedora empresarial, em que são atores os enfermeiros que atuam como liberais ou empresários, em clínicas, consultórios e empresas a possibilidade de ampliação do acesso a saúde por meio do oferecimento de alternativas de cuidado e serviços a parcela da população que possui recursos com proteção ao risco financeiro a que estão sujeitos pela especulação do mercado de saúde.

Identificam-se diversos fatores que encorajam o empreendedorismo de negócios: o envelhecimento populacional, as gerações dispostas a pagar pela saúde, a terceirização e a inserção de serviços suplementares não cobertos pelos serviços públicos ou por planos de saúde; bem como as tendências sociais relacionadas a estilos de vida, conveniência e saúde preventiva ${ }^{14}$.

No contexto do SUS, considerando também o Plano Nacional de Saúde 2020-2023 (PNS) ${ }^{7}$, que expõe uma análise de situação de saúde brasileira, tem-se de forma clara o desafio dos serviços existentes e a necessidade de novas iniciativas para ampliar e qualificar o acesso aos bens e serviços de saúde, com interfaces na melhoria das condições de saúde, promoção da equidade e da qualidade de vida dos brasileiros.

Ao que diz respeito ao endereçamento dos desafios dos serviços de saúde, ao empreender e inovar com estratégias que diminuam filas ou reduzam o tempo de espera, que reduzam custos, que qualifiquem e humanizem a assistência prestada, bem como eduquem a população para que contribua com a consolidação do sistema, o pessoal de enfermagem em atuação empreendedora intraorganizacional pode contribuir para a efetivação dos princípios doutrinários do SUS e ODS 3.

Ao considerar o exposto, pode se perceber que a ideia de sustentabilidade por meio do empreendedorismo de Enfermagem não tem somente potencial para atender as demandas básicas de saúde da população, mas de ir além, produzindo riqueza imaterial e material, sendo esta última bastante relevante em um cenário pós pandemia, de reestruturação produtiva ${ }^{15}$. Entretanto, para que isso seja possível profissionais e estudantes necessitam de educação empreendedora, de formação teórica e experiências práticas dos diferentes tipos de empreendedorismo. 
A abordagem do empreendedorismo ao longo da formação inicial e continuada pode contribuir para o fortalecimento de características comportamentais necessárias a atuação empreendedora como a busca de informações, planejamento e monitoramento sistemático, persuasão e rede de $\operatorname{contatos}^{16}$.

\section{CONCLUSÃO}

O empreendedorismo de enfermagem em suas distintas formas e contextos tem potencial de favorecer o alcance do ODS 3 configurando-se como possibilidade de qualificação e ampliação da atuação profissional no mercado de trabalho, do escopo de ações de saúde, bem como para a busca de inovações no cuidado. Como contribuição para a Enfermagem esta reflexão pode ampliar o entendimento da categoria sobre o significado de empreender, auxiliando na desmistificação da ideia de que está somente associado a abertura de um negócio e/ou que se relaciona pouco com o Sistema Único de Saúde.

Uma vez que todos os dezessete objetivos estão interligados, o empreendedorismo promove o ODS3, mas não só sendo também caminho para resolução de problemas do passado da profissão, como a valorização e reconhecimento profissional por meio de fatores higiênicos e motivacionais, e de possibilidades para um futuro sustentável aos serviços e as pessoas, em particular a nova geração de profissionais.

Assim, é pertinente o investimento em ações relacionadas ao ODS 8, que intenciona promover o crescimento econômico sustentado, inclusivo, o fomento ao emprego pleno, produtivo e o trabalho decente para todas e todos. Isto posto, o reconhecimento do trabalho de enfermagem com jornada e remuneração justas precisa ser endereçado e acolhido pela sociedade. Necessita-se também investimento no ODS 4, relacionado a promoção de oportunidades de aprendizagem ao longo da vida, neste caso, com destaque para a educação empreendedora em seus aspectos técnicos e, sobretudo, comportamentais.

Recomenda-se a realização de estudos que identifiquem quais as relações entre as tipologias de empreendedorismo de enfermagem e as metas do ODS 3, 8 e 4, avaliando seu impacto na cobertura universal de saúde, pois a limitação desta reflexão é restringir-se ao ODS 3.

\section{REFERÊNCIAS}

1. Organização das Nações Unidas Brasil (ONU BRASIL). Transformando nosso mundo: a agenda 2020 para o Desenvolvimento Sustentável [site de Internet]. [cited 2020 set 18] 2015. Available from: https://nacoesunidas.org/wpcontent/uploads/2015/10/agenda2030-pt-br.pdf.

2. International Labour Organization. ILO Monitor: Covid-19 and the world of work. Second Edition. Updated estimates and analysis. Genebra (CH):2021.

3. Costa S da S. The pandemic and the labor market in Brazil. Rev Adm Pública [Internet]. 2020 [cited 2020 Jul 26]; 54(4): 969-78. DOI: http://dx.doi.org/10.1590/0034-761220200170x.

4. Mattei L, Heinen VL. Impacto da crise da Covid-19 no mercado de trabalho brasileiro. Brazil. J. Polit. Econ. [Internet]. 2020; [cited 2020 Jul 26]; 40(4):647-68. Available from: https://doi.org/10.1590/0101-31572020-3200.

5. Giovanella L, et al. Universal health system and universal health coverage: assumptions and strategies. Ciênc. saúde colet. [Internet]. 2018 [cited 2020 Aug 20]; 23(6):1763-76. DOI: https://doi.org/10.1590/1413-81232018236.05562018.

6. FIOCRUZ/COFEN. Relatório final da Pesquisa Perfil da Enfermagem no Brasil. Rio de Janeiro (RJ): FIOCRUZ/COFEN; 2017.

7. Ministério da Saúde (Br). Secretaria-Executiva. Subsecretaria de Planejamento e Orçamento. Plano Nacional de Saúde - PNS 2020-2023. Brasília (DF): Ministério da Saúde; 2020.

8. Wennekers Sanders. Entrepreneurshio at Country Level: economic and non-economic determinants. Roterdamm (NL): Erasmus Research Institute; 2006.

9. Coppelli FHS, Erdmann AL, Santos JLG. Entrepreneurship in Nursing: literature review. Rev Bras Enferm. [Internet]. 2019 [cited 2021 Jan 19]; 72(1):301-10. Available from: https://doi.org/10.1590/0034-7167-2017-0523.

10. Kirkman A, Wilkinson J, Scahill S. Thinking about health care differently: nurse practitioners in primary health care as social entrepreneurs. Journal of Primary HealthCare [Internet]. 2018 [cited 2020 Jul 26]; 10 (4): 331-7. Available from: https://doi.org/10.1071/HC18053.

11. Salvage J, White J. Our future is global: nursing leadership and global health. Rev. Latino-Am. [Internet]. 2020; [cited 2020 Jul 26]; 28:e3339. Available from: https://doi.org/10.1590/1518-8345.4542.3339.

12. Machado MH, Oliveira ES, Lemos WR, Lacerda WF, Justino E. Labor market in nursing in the SUS: an approach from the research Nursing Profile in Brazil. Divulg. saúde debate. [Internet]. 2016 [cited 2021 Out 22]; 56:52-69. Available from: http://fiadmin.bvsalud.org/document/view/6c2z5.

13. Backes DS, Ilha S, Weissheimer AS, Halberstadt BMK, Megier ER, Machado R. Socially entrepreneurial activities in nursing: contributions to health/healthy living. Esc Anna Nery [Internet]. 2016 [cited 2020 Apri 14]; 20(1):77-82. Available from: https://www.scielo.br/j/ean/a/PGn4jFbcjskjd7pbVNpLpBb/?format=pdf\&lang=pt.

14. Colichi RMB, Lima SGS e, Bonini ABB, Lima SAM. Empreendedorismo de negócios e Enfermagem: revisão integrativa. Rev. Bras. Enferm. [Internet]. 2019 [cited 2020 Aug 20]; 72 (1):335-45. Available from: https://doi.org/10.1590/0034-7167-2018-0498. 
15. Instituto Brasileiro de Geografia e Estatística (IBGE). O IBGE apoiando o combate à COVID-19 [Internet]. Desocupação, renda, afastamentos, trabalho remoto e outros efeitos da pandemia no trabalho. [cited 2020 set 18] 2020. Available from: https://covid19.ibge.gov.br/pnad-covid/trabalho.php.

16. Chagas SC, Milagres PN, Silva MCR, Cavalcante RB, Oliveira PP de, Santos RC dos. Business entrepreneurship among nurses. Rev. enferm. UERJ [Internet]. 2018 [cited 2020 Aug 20]; 26:e31469. Available from: https://doi.org/10.12957/reuerj.2018.31469. 